\title{
140-220 GHz Imaging Front-end Based on 250 nm InP/InGaAs/InP DHBT Process
}

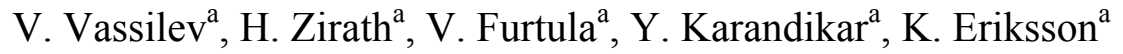 \\ ${ }^{\mathrm{a}}$ Microwave Electronics Laboratory, Department of Microtechnology and Ninoscience - MC2, \\ Chalmers University of Technology, Gothenburg, Sweden
}

\begin{abstract}
This paper presents a pre-amplified detector receiver based on a $250 \mathrm{~nm} \mathrm{InP/InGaAs/InP} \mathrm{double} \mathrm{heterojunction} \mathrm{bipolar}$ transistor (DHBT) process available from the Teledyne scientific. The front end consists of a double slot antenna followed by a five stage low noise amplifier and a detector, all integrated onto the same circuit. Results of measured responsivity and noise are presented. The receiver is characterized through measuring its response to hot (293) and cold (78) K terminations. Measurements of the voltage noise spectrum at the video output of the receiver are presented and can be used to derive the temperature resolution of the receiver for a specific video bandwidth.
\end{abstract}

Keywords: Power detectors, InP, MMIC, DHBT, radiometers, receivers, passive imaging, G-band, responsivity, NEP, flicker noise, remote sensing.

\section{INTRODUCTION}

Applications such as imaging and atmospheric studies require sensitive power detectors. Real-time imaging systems require multi-pixel receivers. Within security imaging, there is an obvious need for cheaper and more compact front-ends that would allow introducing real-time scanning systems at a reasonable price. Passive systems covering W-band (75$110 \mathrm{GHz})$ and D-band (110-170 GHz) are available today and are based on an LNA followed by a power detector [1][4]. The low-noise detector technologies available today include zero-bias Schottky [5] and tunnel diodes [1], [6], [7], however tunnel diodes are not commercially available above the W-band, and Schottky power-detectors, although expensive, are available with high responsivity and low-noise.

To achieve better spatial resolution and more compact scanners, increasing the frequency above $100 \mathrm{GHz}$ is required. Compact and low-cost front ends require a technology where an LNA is integrated with a power detector. Such solutions require the use of HBT or HEMT technologies to integrate a detector on the same MMIC as the LNA. Neither Schottky nor tunnel diodes are suitable for integration with existing low noise HEMT or HBT technologies. Examples of frontends where an LNA and a DC-biased detector are fabricated in the same process and are integrated in one circuit using SiGe processes can be found in [2], [3], and [4]. The drawback of this solution is the need for detector biasing, which implies increased low-frequency noise and requires good quality biasing sources. Usually, LNA-Power detector circuits are designed in such a way that the output level after the LNA is just below the saturation level for the detector, typically -30 to $-40 \mathrm{dBm}$. It is obvious that even for zero-bias detectors pumped at RF power close to saturation $1 / \mathrm{f}$ noise is present.

This paper presents results including measurements of an integrated passive imaging receiver consisting of a double slot antenna, a five stage LNA and a power detector. The circuit is fabricated using a $250 \mathrm{~nm} \mathrm{InP}$ DHBT process from the Teledyne Scientific. The LNA and the detector circuits cover the G-band $(140-220 \mathrm{GHz})$ whereas the band of the receiver is limited by the antenna, which has maximum efficiency around $158 \mathrm{GHz}$. Breakouts of the LNA and the detector circuits are also characterized and results are presented. Measurements of receiver DC response to hot (293) and cold (78) $\mathrm{K}$ are used to calculate receiver noise temperature and thermal sensitivity $(d V / d T)$. Measurements of the voltage noise spectrum at the video output of the receiver are used to extract modeling coefficients for the noise power spectrum density. These coefficients are used to derive the receiver temperature resolution $\Delta T$ for specific video bandwidth. The noise spectrum measurement is also given for hot and cold load terminations and is used to verify the noise temperature and gain of the receiver.

Passive and Active Millimeter-Wave Imaging XVI, edited by David A. Wikner, Arttu R. Luukanen, Proc. of SPIE Vol. 8715, 871502 · C 2013 SPIE · CCC code: 0277-786X/13/\$18 doi: $10.1117 / 12.2018397$

Proc. of SPIE Vol. $8715871502-1$ 


\section{TECHNOLOGY}

The receiver was manufactured in a $250 \mathrm{~nm}$ emitter width InP DHBT process from Teledyne Scientific. The device structure is grown using molecular beam epitaxy on a semi-insulating InP substrate and utilizes a carbon-doped InGaAs base layer with an InP $\mathrm{N}^{-}$collector region. The active devices are passivated with a low-k spin-on dielectric layer of benzocyclobutene (BCB).The unity gain cut-off frequency $\left(f_{t}\right)$ and the power gain cut-off frequency $\left(f_{\max }\right)$ are 350 and $650 \mathrm{GHz}$, respectively while maintaining a common-emitter breakdown voltage $\left(V_{C E O}\right)$ of greater than $4 \mathrm{~V}$. The DHBT process includes thin-film resistor (TFR) with resistance of $50 \Omega$ per square, a MIM capacitor and 4 metal layers (M1 M4). The first layer (M1) contacts the transistor and resistor terminals. Metal-insulator-metal capacitors are formed with a $200 \mathrm{~nm}$ SiN dielectric between M1 and the capacitor metal layer. Three additional interconnect layers are added with $2 \mu \mathrm{m}$ of interlayer BCB dielectric separating the layers. The top metal layer (M4) is $3 \mu \mathrm{m}$ to facilitate the formation of low-loss thin film microstrip line and to accommodate higher current density requirements. A schematic cross-section of the process is shown in Figure 1, more details regarding the process are found in [8].

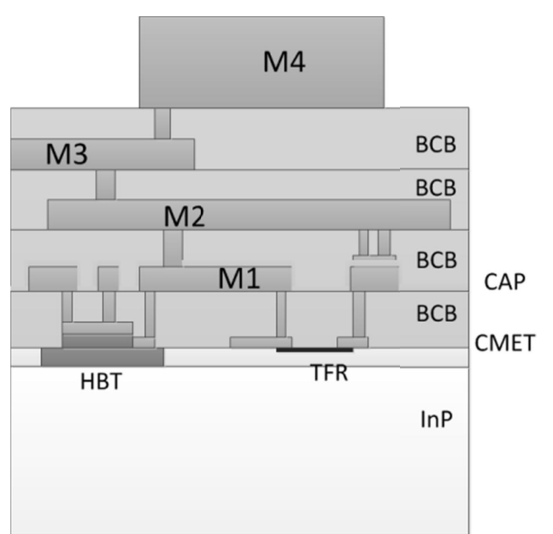

Figure 1 Multilayer structure of the InP DHBT process, for the circuits presented in this paper the thickness of the InP substrate is $100 \mu \mathrm{m}$.

\section{DOUBLE SLOT ANTENNA}

The double slot antenna as described in [9] is implemented in the DHBT Process. The slots are etched in the circuit ground plane using M1 layer while the feeding of the slots is done by microstrip lines and radial stubs using M4 layer. The outputs from both slots are then combined with a T-junction and matched further to $50 \Omega$ by using a quarter wavelength transformer. The layout of the antenna is shown in Figure 2. The slots radiate first in $100 \mu \mathrm{m}$ thick InP substrate followed by a silicon lens beneath it, [10].

The input matching of the slots is designed by placing an infinite Silicon medium below the MMIC for faster simulations. The simulated return loss at the input of the antenna is lower than $-10 \mathrm{~dB}$ over 155-175 GHz. After placing the antenna on a $12 \mathrm{~mm}$ diameter Si lens, the antenna bandwidth is reduced and the resonance frequency is shifted to a lower frequency. The resultant return loss together with the return loss in the case of infinite Si medium is plotted in Figure 3; the lens includes an anti-reflection coating. 


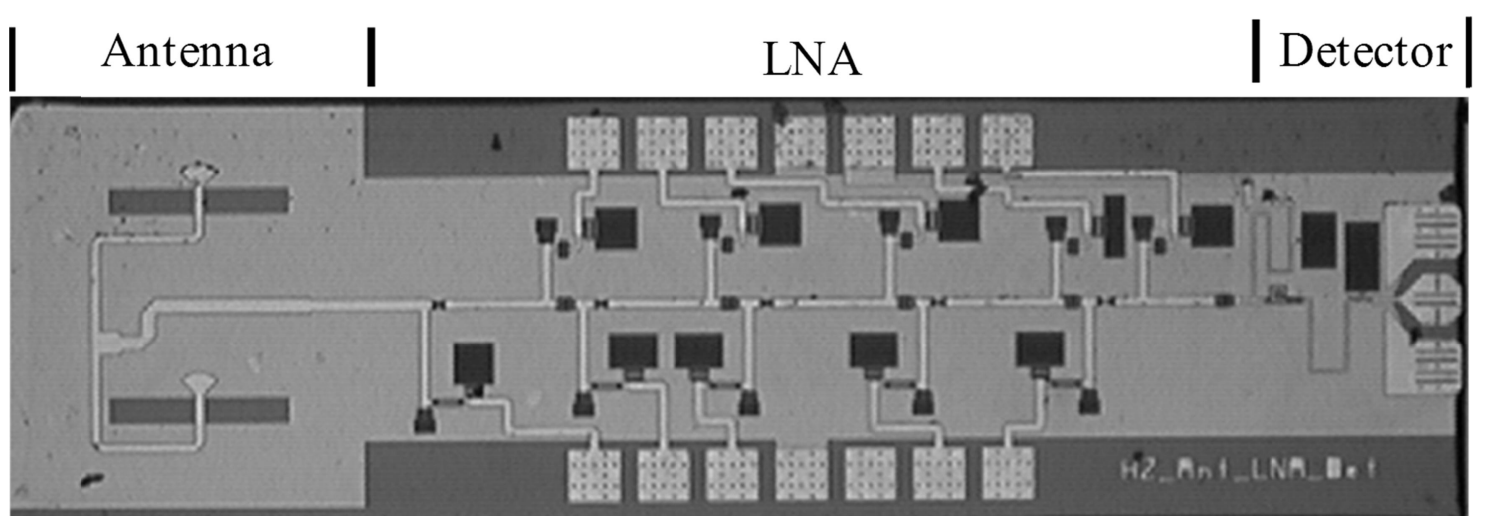

Figure 2 Layout of the receiver. A double slot antenna is followed by a five stage LNA and a detector, the chip size is $2140 / 625 \mu \mathrm{m}$.

The antenna with Si Lens has directivities in the range of $22-24 \mathrm{dBi}$ over the band of interest. The $\varphi=45^{\circ}$ pattern of the complete structure is shown in Figure 3. The ohmic efficiency of the antenna considering dielectric and conductive losses is $\sim-1.7 \mathrm{~dB}$ at $160 \mathrm{GHz}$.
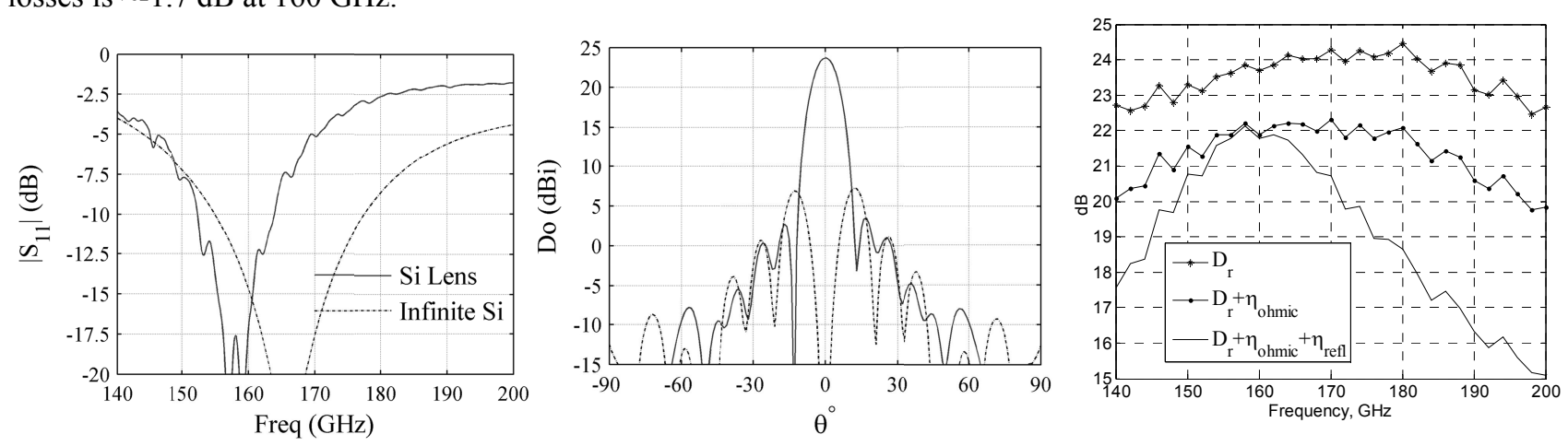

Figure 3 Antenna reflection coefficient, copolar/crosspolar patterns at $160 \mathrm{GHz}$ and directivity and gain.

\section{LNA}

A 5-stage amplifier was designed and optimized for broad band gain between 110 and $200 \mathrm{GHz}$. The emitter lengths of the transistors are selected as 2, 3,3,6, and $10 \mu \mathrm{m}$. Transistors are biased with constant current at the bases, achieving a collector current density of $5-10 \mathrm{~mA} / \mu \mathrm{m}^{2}$, which is close to maximum gain bias. The base current $I_{b}$ is applied separately to each stage and have typical values of $150 \mu \mathrm{A}, 300 \mu \mathrm{A}, 350 \mu \mathrm{A}, 400 \mu \mathrm{A}, 550 \mu \mathrm{A}$ for stages 1 to 5 .

The measurements on the LNA are performed using PNA N5250C equipped with OML frequency extension modules for the frequencies in the range from $140 \mathrm{GHz}$ to $225 \mathrm{GHz}$. For measurements below $110 \mathrm{GHz}$ a, standard PNA in combination with W-band frequency extenders were used. The circuits were measured attached on top of a silicon piece and S-parameters measurements are shown in Figure 4. 

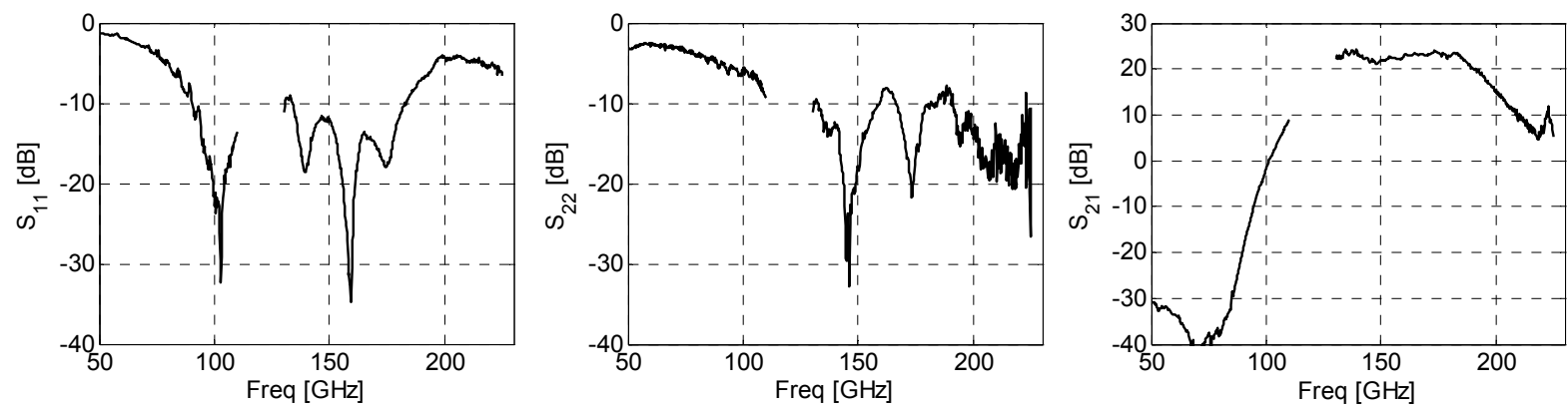

Figure 4 Measured S-parameters.

\section{DETECTOR}

The detector is implemented by using the base-emitter junction of a DHBT with sizes $0.25 \mathrm{x} 2 \mu \mathrm{m}$. The bias current is driven through the base-emitter junction creating a DC voltage component at the base $V_{b 0}$. The collector port is not short circuited with the base and a DC voltage component $V_{c 0}$ results from collector self-biasing. The DC component of the detected voltage can be measured either at the base or at the collector terminals. For the measurements of the detector breakout circuit the collector port was used to measure the peak to peak voltage of the detected waveform (as shown in Figure 5). For the radiometric measurements detected-voltage reading from the base port was provided by the current source (Keithley 2425 source meter) while the collector port was used to take the spectrum of the detected voltage. In this configuration, although the transistor is used as three-terminal device, the collector port is not actively biased and therefore the detector is essentially a passive diode detector, where the base-emitter junction non-linearity is used for detection.



Figure 5 Detector circuit and the setup used to measure responsivity of the detector breakout MMIC. The detector is AC coupled to a lock-in-amplifier. Pulse modulation at $1 \mathrm{kHz}$ was used to create the $\mathrm{AC}$ waveform.

Analysis of the measured IVC and the extracted, from measurements, series resistance $R s$ and junction capacitance $C_{b e}\left(V_{b e}\right)$ shows that the responsivity peaks at $V_{b e}=0.57 \mathrm{~V}$ (bias current of $1 \mu \mathrm{A}$ ) and has a value of $10500 \mathrm{~V} / \mathrm{W}$ at 160 $\mathrm{GHz}$. This value of responsivity does not include losses in the circuit. The measured maximum responsivity is $9700 \mathrm{~V} / \mathrm{W}$ at $160 \mathrm{GHz}$, and takes place at $1 \mu \mathrm{A}$ bias current as expected, the video resistance $d V_{b e} / d I_{b e}$ at this bias is $27 \mathrm{k} \Omega$. Results of responsivity measurements of a breakout of the detector circuit for three RF frequencies are shown in Figure 7.

\section{ANTENNA - DETECTOR}

Responsivity of the detector circuit was also measured when integrated together with the double slot antenna. The antenna-detector MMIC chip is mounted on a $12 \mathrm{~mm}$ diameter Si Lens and the lens is further placed on an elevated bench so that it could receive radiated power from below as shown in Figure 6. The RF is radiated underneath the chip 
using a G-band corrugated horn. The RF source includes a times 6 multiplier chain with a variable attenuator to control the radiated power. The RF is applied with a $1 \mathrm{kHz}$ pulse modulation and $50 \%$ duty cycle. This modulation signal is also used to synchronize the lock-in amplifier with the RF signal generator. The MMIC is biased with $1 \mu \mathrm{A}$ constant current using DC probes. The video output of the detector is fed to the input of the lock-in-amplifier.

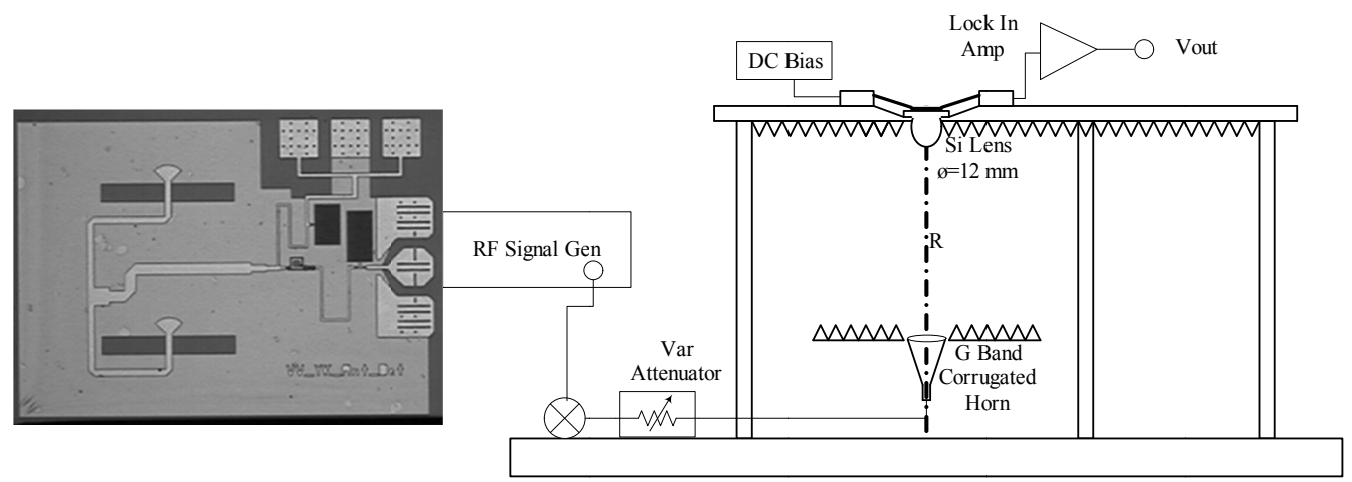

Figure 6 Antenna-detector circuit and responsivity measurement setup for the antenna-detector MMIC.

To estimate the responsivity of the antenna integrated detector, the power before the horn was measured, and the power fed to the detector $P r$ is estimated using the following link budget.

$$
P r=P t+G t+F S L+D
$$

Where $P t$ is the transmitted power, FSL is the free space loss (calculated), Gt is the gain of the transmitting horn, and $D r$ is the receiving antenna directivity (simulated). The reason for using directivity and not the gain of the receiving antenna is that by comparing responsivity with break-out of the detector one can estimate the ohmic losses in the double slot antenna. The detector with integrated antenna was measured at 160,170 and $180 \mathrm{GHz}$ respectively. The measured responsivity of the detector alone (without the antenna) is about 9700 at $160 \mathrm{GHz}$ and it agrees well with the simulated responsivity. For the case of antenna-detector the measured responsivity is less than the detector alone. At $160 \mathrm{GHz}$ the antenna is well matched with the detector (see Figure 3) and the difference in responsivity can be attributed to antenna's ohmic losses (about $0.8 \mathrm{~dB}$ ). The impedance mismatch becomes more significant factor for 170 and $180 \mathrm{GHz}$. At these frequencies, the antenna is highly mismatched and this causes reduction in the measured responsivity, see also Figure 3.
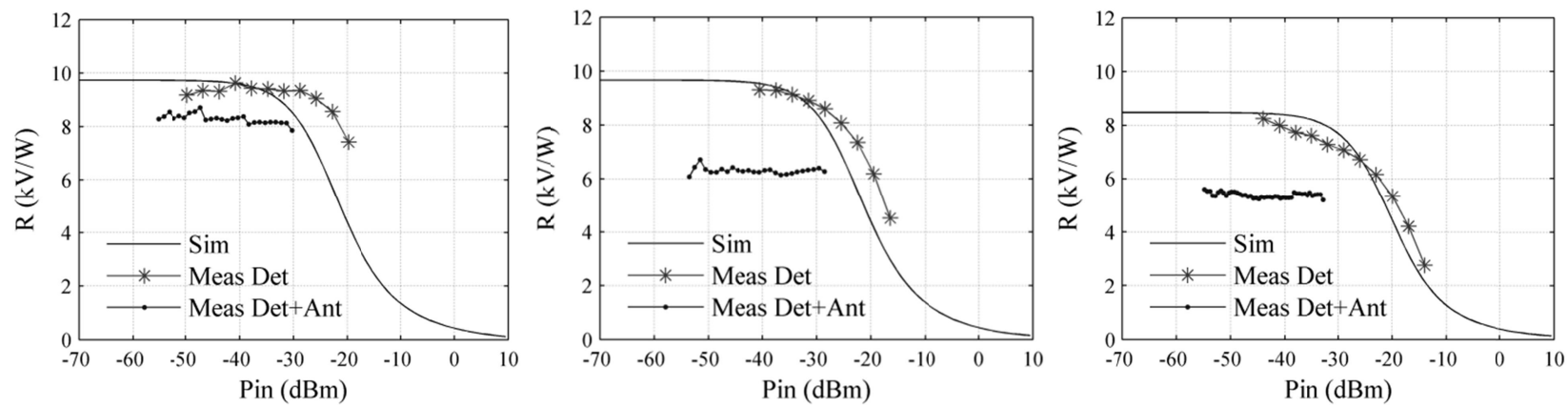

Figure 7 Simulated and measured responsivity of the detector circuit and the antenna-detector circuit at three frequencies: 160,170 and $180 \mathrm{GHz}$.

\section{ANTENNA - LNA - DETECTOR}

The setup used for characterization of the integrated receiver is illustrated in Figure 8. Two sets of measurements are performed: DC response of the detector, and noise voltage spectrum for hot and cold load terminations at the antenna input. The base currents for the LNA were provided by a HP 4156 parameter analyzer, whereas the detector, as before, was biased with a constant current of $1 \mu \mathrm{A}$ provided by a Keithley source meter. No measures were taken to suppress the noise associated with the biasing of the receiver. 

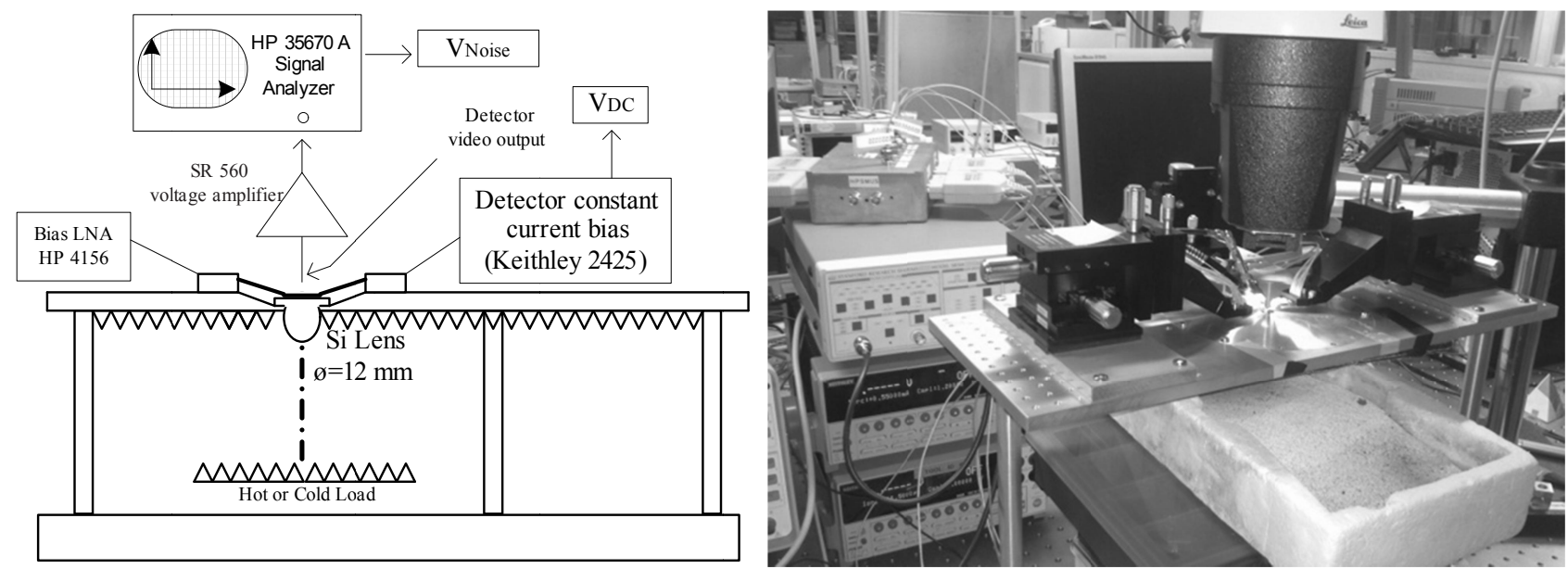

Figure 8 Schematic and a picture of the measurement setup. The detector circuit is biased with a "Keithley 2425" source meter, which is also used to measure the detected voltage. The detector video output is fed to a low-noise video amplifier (SR 560) followed by a FFT signal analyzer (HP 35670A) to retrieve the video noise spectrum. The base currents for the LNA were provided by a HP 4156 parameter analyzer.

The DC measurements are performed by reading values of the detected voltage from the source meter used to provide current bias for the detector. From this measurement the following radiometer parameters are derived: receiver Y-factor and noise temperature, radiometer sensitivity $d V / d T$, and gain. Examples of time series of detected voltages are shown in Figure 9 for two bias points specified in Table 1.
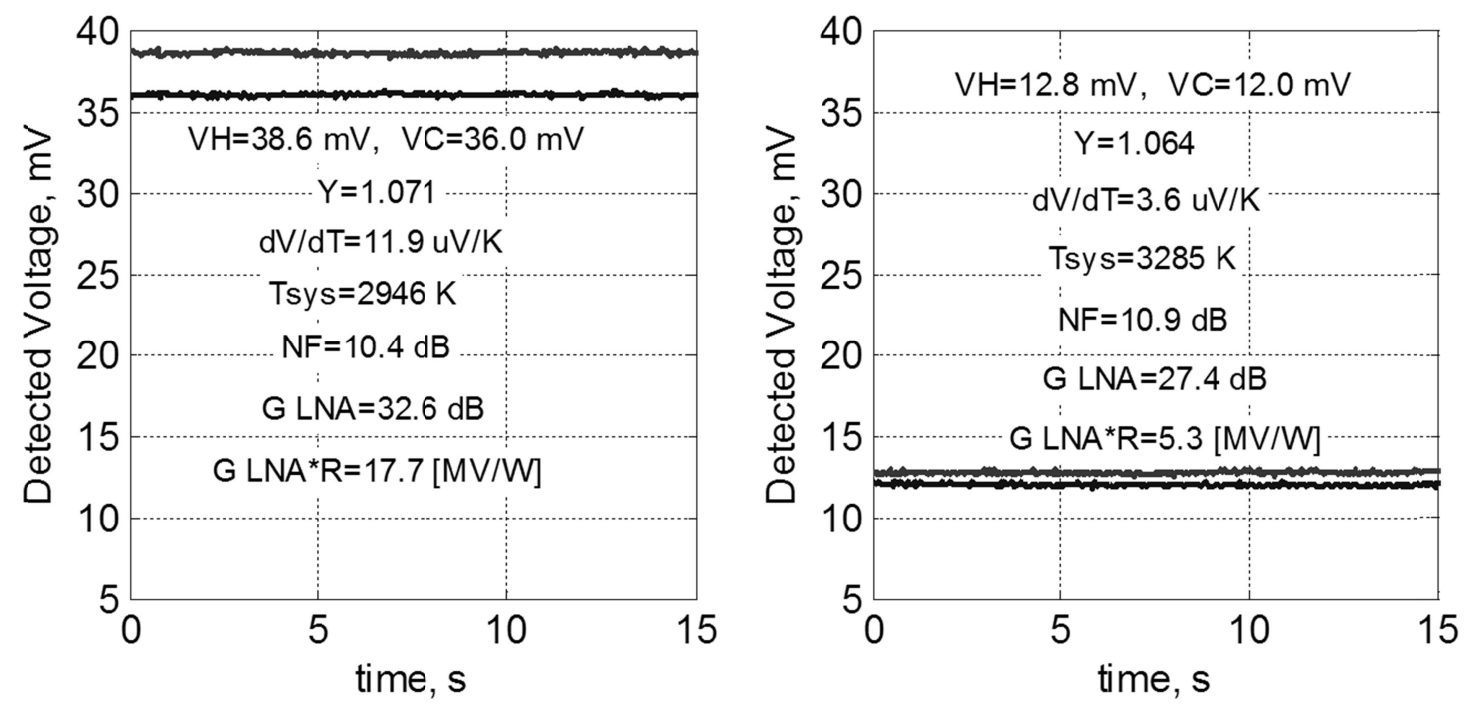

Figure 9 Time series of detected DC voltages for Hot/Cold load terminations and for the two bias points. To the left: bias point a); To the right bias point b), The simulated NF values are 11.5 and 11.7.

$$
\frac{\Delta V}{\Delta T}=\frac{\left(V_{D C}^{\text {hot }}-V^{\text {cold }}{ }_{D C}\right)}{(294-78)}=G_{L N A} \cdot R \cdot k \cdot B_{R F}, \quad\left[\frac{V}{K}\right]
$$

From eq.(2) the thermal sensitivity is $11.9 \mu \mathrm{V} / \mathrm{K}$ and $3.6 \mu \mathrm{V} / \mathrm{K}$ for bias point a) and b). The gain of the LNA can be extracted from the measured DC voltages and LNA noise temperature, but requires assumptions on the RF bandwidth and detector responsivity. The simulated antenna bandwidth is between $145-176 \mathrm{GHz}$ (3dB drop in efficiency), whereas the LNA has $80 \mathrm{GHz}$ bandwidth ( $3 \mathrm{~dB}$ drop in $\mathrm{S}_{21}$ ). The total responsivity of the radiometer, which is a product of the 
LNA gain and the detector responsivity is $17.7 \mathrm{MV} / \mathrm{W}$ for bias a) and 5.3 MV/W for bias b). Considering the measured responsivity of the break-out circuit of the detector at $160 \mathrm{GHz}$ being $9700 \mathrm{~V} / \mathrm{W}$, the LNA gain can be de-embedded and is $32 \mathrm{~dB}$ for bias point a). This is a higher gain considering the LNA on-wafer measurements for the same bias point $(\mathrm{S} 21=24 \mathrm{~dB}$ at $160 \mathrm{GHz})$ and the simulated gain $30 \mathrm{~dB}$.

\begin{tabular}{|c|c|c|c|c|c|c|c|}
\hline Item & $I_{b 1}, \mu A$ & $I_{b 2}, \mu A$ & $I_{b 3}, \mu A$ & $I_{b 4,}, \mu A$ & $I_{b 5}, \mu A$ & $V_{c}, V$ & $I_{d e t} \mu A$ \\
\hline Emitter L, $\mu \mathrm{m}$ & 2 & 3 & 3 & 6 & 10 & & \\
\hline Bias a) & 150 & 300 & 350 & 400 & 550 & 1.5 & 1 \\
\hline Bias b) & 80 & 150 & 300 & 400 & 550 & 1.5 & 1 \\
\hline
\end{tabular}

Table 1 Bias points for the LNA stages used in the noise gain characterization. The collector currents are $\beta$ times higher where $\beta$ is 23 for the first stage and about 25 for stages 2 to 5 .

Noise equivalent power (NEP) is a figure of merit for power detectors and can be used to calculate the temperature resolution of the radiometer. The NEP is defined as the noise power at the input of the detector that produces SNR at the output equal to one. The NEP is the input signal power that produces detected voltage equal to the measured noisevoltage $V n$. In this work the NEP is calculated from measurements of $V n$ at the output of the detector and thus is related to the bandwidth over which $V n$ is measured. To make possible comparison between detectors, $V n$ is measured over a bandwidth of $1 \mathrm{~Hz}$ and its value is given in units of $\mathrm{V} / \sqrt{\mathrm{Hz}}$. Because of the presence of $1 / \mathrm{f}$ noise it is useful to present the NEP for a specific video frequency. The NEP is calculated as:

$$
N E P=\frac{V_{n}}{R} \quad[\mathrm{~W} / \sqrt{\mathrm{Hz}}]
$$

The NEP of the detector and the receiver are shown in Figure 10 and can be used to calculate the temperature resolution of the radiometer (eq.(5).
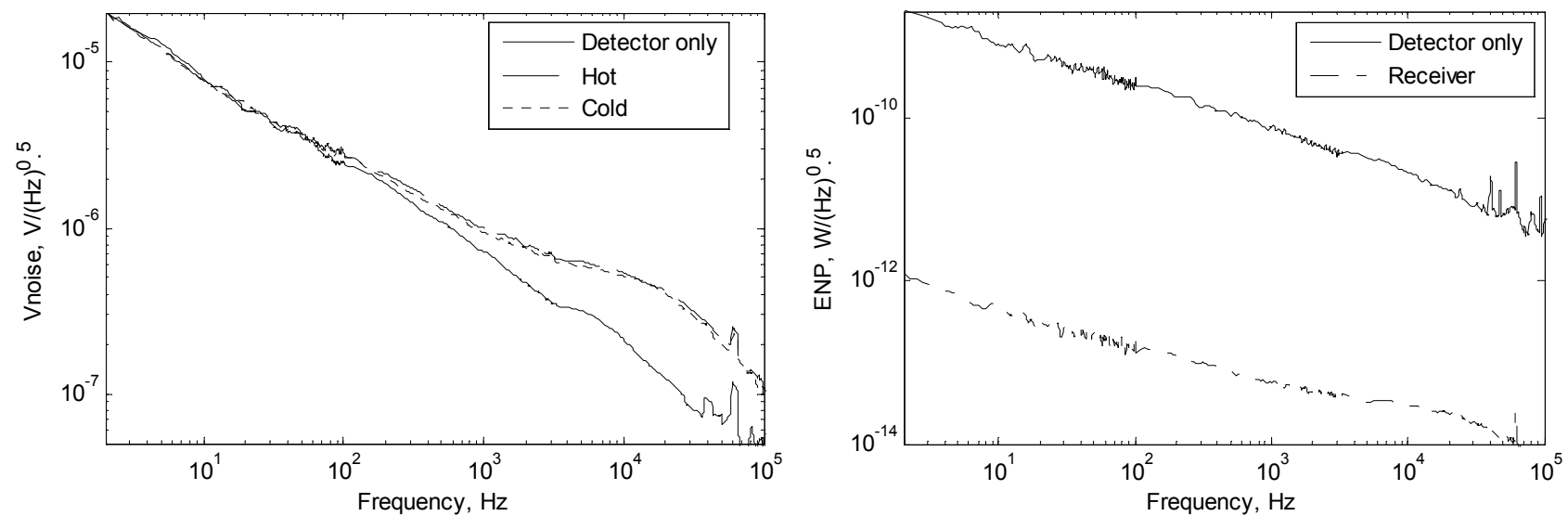

Figure 10 Measured noise voltage and the calculated ENP at $160 \mathrm{GHz}$ for the receiver at bias point a) and for the detector only. Responsivity of $17.7 \mathrm{MV} / \mathrm{W}$ is considered for the receiver and $9700 \mathrm{~V} / \mathrm{W}$ for the detector.

Since the receiver responsivity is considerably higher for the bias point a) this bias was chosen for the calculation of the NEP and for the rest of the measurements presented below.

The noise voltages vs. video frequency are measured for both hot/cold terminations and for the case when the LNA is switched off (detector noise only), the result is shown in Figure 10. The noise voltage measurement can also be used to verify the Y-factor derived using the DC measurements from Figure 9, as the ratio of $\Delta T_{H o t} / \Delta T_{\text {Cold }}$ from (4) is by definition the Y-factor of the system and is also equal to the ratio of the noise voltages $\Delta T_{\text {Hot }} / \Delta T_{\text {Cold }}=V_{H o t}{ }^{n} / V_{\text {Cold }}{ }^{n}$. 


$$
\Delta T_{H o t}=\frac{T_{S Y S}+T_{H o t}}{\sqrt{B_{R F} \cdot \tau}} ; \Delta T_{\text {Cold }}=\frac{T_{S Y S}+T_{\text {Cold }}}{\sqrt{B_{R F} \cdot \tau}}
$$

The Y-factor calculated from the measured noise spectrum and shown in Figure 11 is consistent with the DC measurement from Figure 9.

Calculation of the receiver temperature resolution requires knowledge of the type of calibration and video bandwidth $\Delta f_{v i d} f_{v}^{\text {Max }}-f_{v}^{\text {Min }}$. The maximum video frequency $f_{v}^{\text {Max }}$ is inversely proportional to the integration time $\tau$ whereas the minimum video frequency $f_{v}^{M i n}$ is related to how often the radiometer is calibrated. The choice of video bandwidth is a part of the system design and depends on the type of radiometer and the application. The temperature resolution can be calculated by using equation (5) and requires integration of either the noise voltage or NEP.

$$
\Delta T=\frac{V^{n} \cdot \sqrt{B_{V I D}}}{G_{L N A} \cdot R \cdot k \cdot B_{R F}}=\frac{V^{n} \cdot \sqrt{B_{V I D}}}{\Delta V / \Delta T}=\frac{N E P \cdot \sqrt{B_{V I D}}}{k \cdot B_{R F}}
$$

A convenient alternative is given in [1] where the noise power spectrum at video frequencies is modeled and model coefficients are extracted. The equation used to model the noise power spectrum at video frequencies is given by:

$$
S v(f)=\frac{S w+K^{\prime} \frac{v^{2}}{f^{a}}}{1+\left(\frac{f}{f c}\right)^{2}}
$$

Where $a$ is a constant close to $1, K^{\prime}$ is a dimensionless constant for $a=1, V$ is the detected DC voltage, $f$ is the video frequency and $f_{c}$ is the video cut-off frequency. The video cut-off frequency is defined by the relatively high detector video impedance in combination with the input capacitance of the video amplifier $(25 \mathrm{pF})$ and the detector capacitance at the video output $(2 \mathrm{pF}) . S w$ is the white noise spectrum density at the output of the detector, which is a product of the Boltzmann constant, the LNA noise temperature (in addition to the scene temperature) and the total responsivity of the radiometer. In Figure 11 the measured power spectrum is given for both terminations and for the case of LNA switched off.
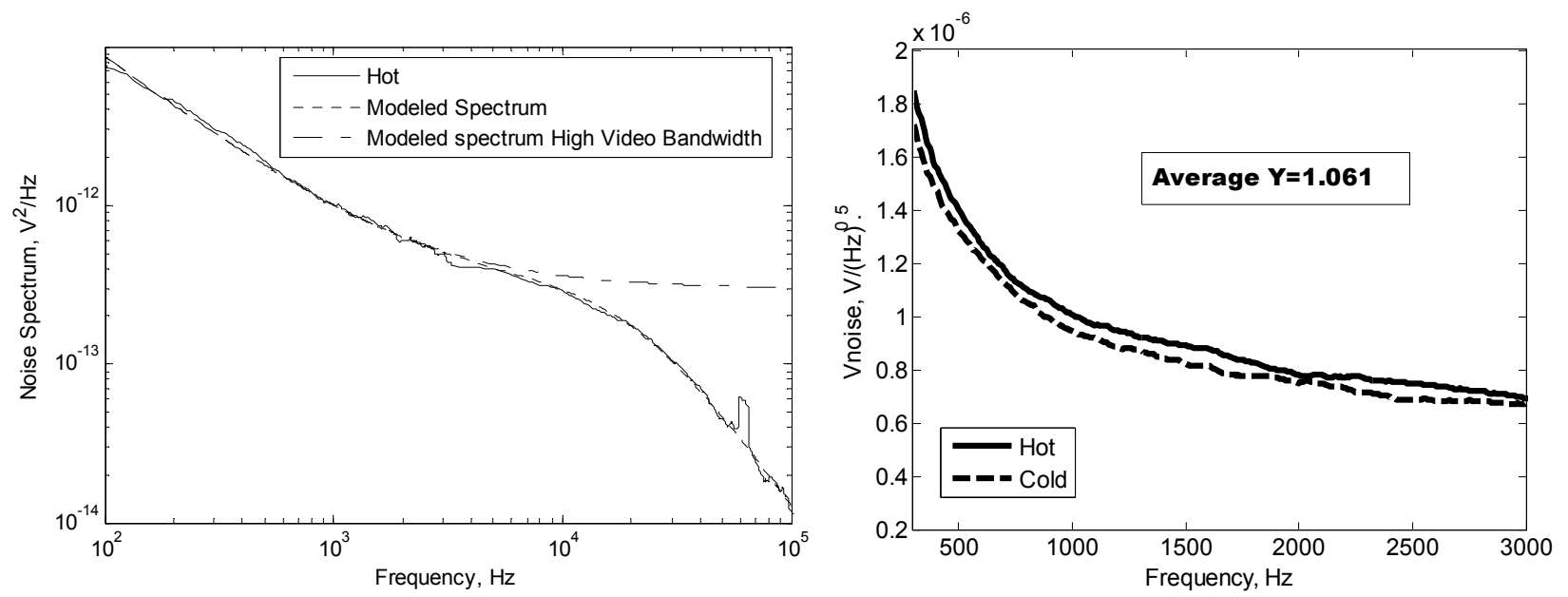

Figure 11 Left: The noise power spectrum measured at the detector output for hot load and the modeled spectrum. The plot also shows the spectrum for the case of broad video band not limited by the voltage amplifier. The measurement is performed in three bands $2 \mathrm{~Hz}-100 \mathrm{~Hz}, 100 \mathrm{~Hz}-3 \mathrm{kHz}$ and $3 \mathrm{kHz}-100 \mathrm{kHz}$. The plots are taken with 30 spectrum averages in the first band and 50 averages for the second and the third bands. Right: Noise voltages at the output of the detector between $300-3 \mathrm{kHz}$ and the ratio of the noise voltages for bias point a) corresponding to Y-factor. 
The model parameters from eq. (6) are used to fit a curve to the measured spectrum. The estimated model parameters are summarized in Table 2.

\begin{tabular}{|l|c|c|c|c|c|}
\hline$S w$ & $f_{c}$ & $K^{\prime}$ & $K$ & $a$ & $V$ \\
\hline $3 e-13$ & $f_{\mathcal{C}}=22 \mathrm{kHz}$ & $7 * 10^{-7}$ & $8.8 * 10^{-7}$ & 1.07 & $38.6 \mathrm{mV}$ \\
\hline
\end{tabular}

Table 2 The model parameters used to fit the measured noise power spectrum from Figure 11 for hot load termination and for bias point a). $K$ is the modified $K^{\prime}$ constant according to [1].

The noise spectrum parameters and the video bandwidth can then be used to calculate $\Delta T$ according to eq. (7), where constant $K$ is the modified $K^{\prime}$ constant for the case of $a \neq 1$ for more details look at reference [1]:

$$
\Delta T=T_{s y s} \sqrt{\frac{S_{w} \cdot \Delta f_{v i d}}{V^{2}}+K \cdot \ln \left(1+\frac{\Delta f_{v i d}}{f_{v i d}^{\min }}\right)}
$$

Using the receiver parameters presented in Table 2 and for an imaging system where a single receiver is scanning 32 pixels in $0.1 \mathrm{~s}$ (the video bandwidth is confined within 5 to $160 \mathrm{~Hz}$ ) the calculated temperature resolution from eq. (7) is $5.7 \mathrm{~K}$.

\section{CONCLUSION}

A radiometer receiver centered at $160 \mathrm{GHz}$ is demonstrated using $250 \mathrm{~nm} \mathrm{InP} / \mathrm{InGaAs} / \mathrm{DHBT}$ process. The receiver contains a double slot antenna, an LNA and a detector all integrated on one chip. The performance of the radiometer is presented in addition to results from on-wafer characterization of the receiver building blocks. The radiometer is a result of connecting three circuits designed for other applications. For example the LNA is optimized for wide bandwidth of about $80 \mathrm{GHz}$. Even though wide bandwidth is a figure of merit in passive systems, the substrate integrated antenna together with the lens are restricting the RF bandwidth of the radiometer to about $31 \mathrm{GHz}$. Therefore the wide bandwidth of the LNA is not contributing to better temperature resolution. A better optimized system would have the LNA band matching the achievable band from the antenna and optimized for lower noise temperature within this band. We believe that $8.5 \mathrm{~dB}$ noise figure is possible at $200 \mathrm{GHz}$ with this process. The expected temperature resolution for a single receiver scanning 32 pixels in $0.1 \mathrm{~s}$ is estimated to be about $5.7 \mathrm{~K}$.

We conclude that, for this particular design, the thermal resolution of the radiometer is not limited by the LNA noise, but limited by the noise in the detector and therefore additional LNA gain is needed to improve the resolution. No measures to cancel possible noise contributions from bias supplies were considered during the measurements presented in this paper. No optimization of detector or LNA bias was used to enhance the performance of the receiver.

\section{ACKNOWLEDGMENT}

Authors would like to acknowledge Miguel Urteaga from Teledyne Scientific and Imaging, for the very useful discussion on noise in bipolar transistors.

\section{REFERENCES}

[1] Lynch J. J., Moyer H.P., Schaffner J. H., Royter Y., Sokolich M., Hughes B., Yoon Y. J., Schulman J. N., "Passive Millimeter-Wave Imaging Module With Preamplified Zero-Bias Detection", IEEE Trans. on Microw. Theory Tech., Vol. 56, pp. 1592-1600 (2008).

[2] May J. W., Rebeiz G. M., "Design and Characterization of W-Band SiGe RFICs for Passive Millimeter-Wave Imaging", IEEE Transactions on Microwave Theory and Techniques, Volume: 58 , Issue: 5 , Page(s): 1420 - 1430 (2010).

[3] Dacquay E., Tomkins A., Yau K. H. K., Laskin E., Chevalier P., Chantre A., Sautreuil B. and Voiginescu S. P., "Dband total power radiometer performance optimization in a SiGe HBT technology," IEEE Trans. Microw. Theory Tech., (2012).

[4] Gilreath L., Jain V., Heydari P., "Design and Analysis of a W-Band SiGe Direct-Detection-Based Passive Imaging Receiver", IEEE Journal of Solid-State Circuits, vol. 46, pp. 2240 - 2252 (2011). 
[5] Neidert R. and Binari S., "Millimeter-wave planar InP Schottky diodes and their small-signal equivalent circuit" IEEE Trans. Microw. Theory Tech., vol. 37, no. 11, pp. 1694-1698, Nov. (1989).

[6] Gabriel W., "Tunnel-diode low-level detection," IEEE Trans.Microwave Theory and Tech., vol. MTT-15, no. 10, pp. 538-553, Oct. (1967).

[7] Kazemi H., Nagy G., Tran L, Grossman E., Brown E. R., Gossard A. C., Boreman G. D., Lail B, Young A. C., Zimmerman J. D., "Ultra sensitive ErAs/InAlGaAs direct detectors for millimeter wave and $\mathrm{THz}$ imaging applications", IEEE/MTT-S International Microwave Symposium, (2007).

[8] Urteaga M., Seo M., Hacker J., Griffith Z., Young A., Pierson R., Rowell P., Skalare A., and Rodwell M. J. W., "InP HBT integrated circuit technology for terahertz frequencies," in Proc. IEEE Compound Semicond. Integr. Circuit Symp. (CSICS), (2010).

[9] Yu Y., Karandikar Y., Gunnarsson S.E., Motlagh B. M., Cherednichenko S.; Kallfass I., Leuther P., Zirath H., "Monolithically Integrated 200-GHz Double-Slot Antenna and Resistive Mixers in a GaAs-mHEMT MMIC Process", IEEE Transactions on Microwave Theory and Techniques, Vol. 59, (2011).

[10] Filipovic D. F., Gearhart S. S., and Rebeiz G. M., "Double-Slot Antennas on Extended Hemispherical and Elliptical Silicon Dielectric Lenses", IEEE Transactions on Microwave Theory and Techniques, Vol. 41, No. 10, (1993).

[11] Racette P. E., "Radiometer Design Analysis Based Upon Measurement Uncertainty", PhD dissertation, Faculty of The School of Engineering and Applied Science of The George Washington University, (2005). 